Research Article

\title{
Numerical High-Order Model for the Nonlinear Elastic Computation of Helical Structures
}

\author{
Fatima Boussaoui, Hassane Lahmam (D), and Bouazza Braikat \\ Engineering and Materials Laboratory (LIMAT), Faculty of Sciences Ben M'Sik, Hassan II University of Casablanca, BP 7955, \\ Sidi Othmane, Casablanca, Morocco \\ Correspondence should be addressed to Hassane Lahmam; hassane.lahmam@univh2c.ma
}

Received 21 November 2020; Revised 18 January 2021; Accepted 23 February 2021; Published 18 March 2021

Academic Editor: Angelos Markopoulos

Copyright (c) 2021 Fatima Boussaoui et al. This is an open access article distributed under the Creative Commons Attribution License, which permits unrestricted use, distribution, and reproduction in any medium, provided the original work is properly cited.

In this work, we propose a high-order algorithm based on the asymptotic numerical method (ANM) for the nonlinear elastic computation of helical structures without neglecting any nonlinear term. The nonlinearity considered in the following study will be a geometric type, and the kinematics adopted in this numerical modeling takes into account the hypotheses of Timoshenko and de Saint-Venant. The finite element used in the discretization of the middle line of this structure is curvilinear with twelve degrees of freedom. Using a simple example, we show the efficiency of the algorithm which was carried out in this context and which resides in the reduction of the number of inversions of the tangent matrix compared to the incremental iterative algorithm of Newton-Raphson.

\section{Introduction}

Helical structures, particularly springs, are considered to be the most used mechanical elements in several industries such as aeronautics, automotive, and civil engineering. Due to their reversible elastic deformability, springs can store or release energy when they are subjected to different loads such as normal force, torque, or both $[1,2]$.

Numerical modeling of the linear elastic behavior of helical springs has been studied by many searchers. Taktak et al. [3] proposed a mixed hybrid formulation, including shear effects, based on the use of a curvilinear finite element with two nodes and six degrees of freedom per node. Dammak et al. [4] have shown that it is possible to obtain the distribution of the various generalized stresses along the middle line of the helical spring with great precision by using a single curvilinear finite element with twelve degrees of freedom. Taktak et al. [5, 6] also used this same finite element for the calculation of the natural frequencies and the dynamic response of a simple or assembled helical spring. Other researchers [7-11] have used theoretical and numerical methods for the dynamic behavior study as well as the buck- ling of coil springs. However, most of these studies are limited to the linear elastic case.

The objective of this work is to propose a high-order algorithm based on the asymptotic numerical method (ANM) [12-17] to calculate the displacements and rotations at a point of the middle line of a helical structure. The originality of our numerical approach is to calculate these unknowns without neglecting any nonlinear term of the strain. The finite element used in our numerical approach is of curvilinear type, defined along the middle line of the structure considered $[18,19]$. The kinematics adopted in our theoretical formulation takes into account the hypotheses of Timoshenko and de Saint-Venant [3-6]. The efficiency of the proposed algorithm lies in the reduction of the number of inversions of the tangent matrix compared to the incremental iterative algorithm of Newton-Raphson [20, 21].

\section{Problem Modeling}

We consider a helical structure of a circular section having a middle radius $R$, a constant pitch $h$, and a length $L$ as shown 
in Figure 1, where $L=\sqrt{R^{2}+(h / 2 \pi)^{2}} \varphi_{\max }$ and $\varphi_{\max }$ is an arbitrary value.

The position vector of each point of the middle line is written, in the Cartesian coordinate system base, as follows:

$$
\overrightarrow{O M}(\varphi)=\left(\begin{array}{c}
R \cos (\varphi) \\
R \sin (\varphi) \\
\frac{h}{2 \pi} \varphi
\end{array}\right)_{\{\vec{i}, \vec{j}, \vec{k}\}},
$$

where $\varphi$ is the polar angle.

The direct orthonormal basis $\{\vec{\tau}, \vec{n}, \vec{b}\}$ of the Frenet coordinate system $R_{\mathrm{F}}$, defined at point $M$, is expressed in the Cartesian basis as follows:

$\vec{\tau}=\frac{1}{\omega}\left(\begin{array}{c}-R \sin (\varphi) \\ R \cos (\varphi) \\ \frac{h}{2 \pi}\end{array}\right)_{\{\vec{i}, \vec{j}, \vec{k}\}} \vec{n}=\left(\begin{array}{c}\cos (\varphi) \\ \sin (\varphi) \\ 0\end{array}\right)_{\{\vec{i}, \vec{j}, \vec{k}\}} \vec{b}=\frac{1}{\omega}\left(\begin{array}{c}\frac{h}{2 \pi} \sin (\varphi) \\ -\frac{h}{2 \pi} \cos (\varphi) \\ R\end{array}\right)_{\{\vec{i}, \vec{j}, \vec{k}\}}$,

where $\omega^{2}=R^{2}+(h / 2 \pi)^{2}$.

The pass matrix from the Cartesian coordinate system to the Frenet coordinate system is given by

$$
[P]=\left[\begin{array}{ccc}
-\frac{R}{\omega} \sin (\varphi) & \frac{R}{\omega} \cos (\varphi) & \frac{h}{2 \pi \omega} \\
\cos (\varphi) & \sin (\varphi) & 0 \\
\frac{h}{2 \pi \omega} \sin (\varphi) & -\frac{h}{2 \pi \omega} \cos (\varphi) & \frac{R}{\omega}
\end{array}\right]
$$

The derivatives of these vectors with respect to the curvilinear abscissa are given by

$$
\begin{aligned}
& \frac{d \vec{\tau}}{d s}=\rho_{1} \vec{n}, \\
& \frac{d \vec{b}}{d s}=-\rho_{2} \vec{n}, \\
& \frac{d \vec{n}}{d s}=\rho_{2} \vec{b}-\rho_{1} \vec{\tau},
\end{aligned}
$$

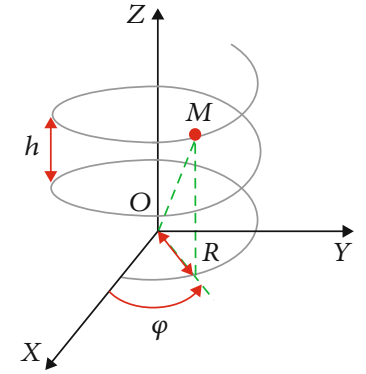

Figure 1: Geometric description of the helical structure.

where $\rho_{1}$ and $\rho_{2}$ designate, respectively, the radius of curvature and the radius of torsion, defined by

$$
\begin{aligned}
& \rho_{1}=\frac{R}{\omega^{2}}, \\
& \rho_{2}=\frac{h}{2 \pi \omega^{2}},
\end{aligned}
$$

with $d s=\sqrt{R^{2}+(h / 2 \pi)^{2}} d \varphi$.

Using the Timoshenko and de Saint-Venant hypotheses [3-6], the components of the displacement vector at a point $P$ in the cross section are written in the Frenet coordinate system as follows:

$$
\left\{\begin{array}{l}
U_{1}\left(s, X_{2}, X_{3}\right)=u(s)-X_{2} \theta^{3}(s)+X_{3} \theta^{2}(s), \\
U_{2}\left(s, X_{2}, X_{3}\right)=v(s)-X_{3} \theta^{1}(s), \\
U_{3}\left(s, X_{2}, X_{3}\right)=w(s)+X_{2} \theta^{1}(s),
\end{array}\right.
$$

where $X_{2}$ and $X_{3}$ are the Cartesian coordinates in the cross section. $u(s), v(s), w(s), \theta^{1}(s), \theta^{2}(s)$, and $\theta^{3}(s)$ are the displacements and rotations at a point in the middle line as shown in Figure 2.

The gradient of the displacements at each point of the cross section is given by

$$
[\bar{\nabla}(\vec{U}(P))]_{R_{\mathrm{F}}}=\left[\begin{array}{ccc}
\frac{1}{\left(1-\rho_{1} X_{2}\right)}\left(\frac{\partial U_{1}}{\partial s}-\rho_{1} U_{2}+\rho_{1} X_{3} \frac{\partial U_{1}}{\partial X_{2}}-\rho_{2} X_{2} \frac{\partial U_{1}}{\partial X_{3}}\right) & \frac{\partial U_{1}}{\partial X_{2}} & \frac{\partial U_{1}}{\partial X_{3}} \\
\frac{1}{\left(1-\rho_{1} X_{2}\right)}\left(\frac{\partial U_{2}}{\partial s}+\rho_{1} U_{1}-\rho_{2} U_{3}+\rho_{2} X_{3} \frac{\partial U_{2}}{\partial X_{2}}-\rho_{2} X_{2} \frac{\partial U_{2}}{\partial X_{3}}\right) & \frac{\partial U_{2}}{\partial X_{2}} \frac{\partial U_{2}}{\partial X_{3}} \\
\frac{1}{\left(1-\rho_{1} X_{2}\right)}\left(\frac{\partial U_{3}}{\partial s}+\rho_{2} U_{2}+\rho_{2} X_{3} \frac{\partial U_{3}}{\partial X_{2}}-\rho_{2} X_{2} \frac{\partial U_{3}}{\partial X_{3}}\right) & \left.\frac{\partial U_{3}}{\partial X_{2}} \frac{\partial U_{3}}{\partial X_{3}}\right]_{R_{\mathrm{F}}}
\end{array} .\right.
$$




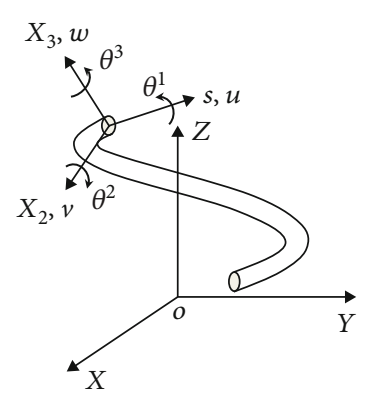

Figure 2: Displacements and rotations of the middle line in the Frenet coordinate system.

The Green-Lagrange strain vector is given by the following formula:

$$
[\gamma(P)]_{R_{\mathrm{F}}}=\frac{1}{1-\rho_{1} X_{2}}\left[\begin{array}{ccc}
\gamma_{11} & \gamma_{12} & \gamma_{13} \\
\gamma_{12} & 0 & 0 \\
\gamma_{13} & 0 & 0
\end{array}\right]_{R_{\mathrm{F}}},
$$

where $\gamma_{11}=e_{1 t}+X_{2}^{2} e_{t 3}+X_{3}^{2} e_{t 2}-X_{2} \chi_{b}+X_{3} \chi_{n}+X_{2} X_{3} \chi_{b n 1}+$ $X_{2}^{3} \chi_{b n 2}+X_{2} X_{3}^{2} \chi_{b n 3}+X_{2}^{2} X_{3} \chi_{b n 4}, \quad \gamma_{12}=\gamma_{t n}+X_{2} \chi_{t 1}-X_{3} \chi_{t 2}$, and $\gamma_{13}=\gamma_{t b}+X_{2} \chi_{t 3}+X_{3} \chi_{t 4}$, with $e_{t 1}, e_{t 2}$, and $e_{t 3}$ designating the membrane strains; $\chi_{t 1}, \chi_{t 2}, \chi_{t 3}$, and $\chi_{t 4}$ are the strains in the plane $\left(X_{2}, X_{3}\right) ; \chi_{n}$ and $\chi_{b}, \chi_{b n 1}, \chi_{b n 2}$, $\chi_{b n 3}$, and $\chi_{b n 4}$ are, respectively, the strains in the planes $\left(s, X_{3}\right)$ and $\left(s, X_{2}\right)$; and $\gamma_{t n}$ and $\gamma_{t b}$ are the shear strains (see Appendix A).

The stationarity of the potential energy of the studied structure results in

$$
\delta W_{d}(U)-\lambda P_{\text {ext }}(\delta U)=0
$$

where $W_{d}(U)$ represents the elastic strain energy, $P_{\text {ext }}(\delta U)$ is the virtual work of the external forces, and $\lambda$ is the loading parameter.

The expression of the elastic strain energy is given by

$$
W_{d}(U)=\frac{1}{2} \int_{V} \overline{\bar{S}}: \overline{\bar{\gamma}} d V
$$

where $\overline{\bar{S}}$ is the second Piola-Kirchhoff tensor.

The elementary volume at any point of the considered structure is written as

$$
d V=\left(1-\rho_{1} X_{2}\right) d X_{2} d X_{3} d s
$$

The variation of the elastic strain energy is given by the following expression:

$$
\delta W_{d}(U)=\int_{L}\langle\delta \gamma\rangle\{S\} d s
$$

where $\{S\}$ and $\{\gamma\}$ are, respectively, the vectors of the generalized stresses and strains given by

$$
\left\{\begin{array}{l}
\langle\gamma\rangle=\left\langle e_{t 1}, \gamma_{t n}, \gamma_{t b}, \chi_{t 1}, \chi_{t 2}, \chi_{t 3}, \chi_{t 4}, \chi_{n}, \chi_{b}, e_{t 2}, e_{t 3}, \chi_{b n 1}, \chi_{b n 2}, \chi_{b n 3}, \chi_{b n 4}\right\rangle, \\
\langle S\rangle=\left\langle N, T_{n}, T_{b}, M_{1}, M_{2}, M_{3}, M_{4}, M_{n}, M_{b}, M_{5}, M_{6}, M_{7}, M_{8}, M_{9}, M_{10}\right\rangle,
\end{array}\right.
$$

where $N$ is the normal effort, $T_{n}$ and $T_{b}$ are the shearing forces, $M_{n}$ and $M_{b}$ are the bending moments along the transversal axes, $M_{i=1,2,3,4}$ are quantities equivalent to the moment, and $M_{i=5,6,7,8,9,10}$ are additional quantities (see Appendix B).

The gradient vector of generalized displacements is written in the following form:

$$
\{\theta\}^{t}=\left\langle u_{, s}, v_{, s}, w_{, s}, \theta_{, s}^{1}, \theta_{, s}^{2}, \theta_{, s}^{3}, u, v, w, \theta^{1}, \theta^{2}, \theta^{3}\right\rangle
$$

Taking into account the expression (14), the vector of generalized strains can also be written in the following form:

$$
\{\gamma\}=\left([H]+\frac{1}{2}[A(\theta)]\right)\{\theta\}
$$

where $[H]$ is a constant matrix which depends on the radius of curvature and $[A(\theta)]$ is a matrix which depends on the gradient of the unknown of the considered problem (see Appendix C).

The generalized behavior law in nonlinear elasticity is given by

$$
\{S\}=[D]\{\gamma\}
$$

where $[D]$ is the elastic constant matrix which also depends on the radius of curvature of the studied structure (see Appendix D).

By virtue of the previous relations, problem (9) is written:

$$
\left\{\begin{array}{l}
\int_{L}\langle\delta \theta\rangle\left([H]^{t}+[A(\theta)]^{t}\right)\{S\} d s-\lambda P_{\mathrm{ext}}=0, \\
\{S\}=\left([H]+\frac{1}{2}[A(\theta)]\right)\{\theta\} .
\end{array}\right.
$$

\section{Resolution Strategy}

The solution of the nonlinear problem (17) is obtained using a high-order algorithm based on the discretization of the study domain into finite elements, the development of the different unknowns in the Taylor series, and the continuation technique.

3.1. Finite Element Discretization. The discrete form of the problem (17) is obtained by applying the finite element method $[18,19]$. The degrees of freedom of each curvilinear element as shown in Figure 3 are represented by the displacement vector as follows:

$$
\langle q\rangle^{e}=\left\langle u_{i}, v_{i}, w_{i}, \theta_{i}^{1}, \theta_{i}^{2}, \theta_{i}^{3}\right\rangle_{i=1,2}
$$




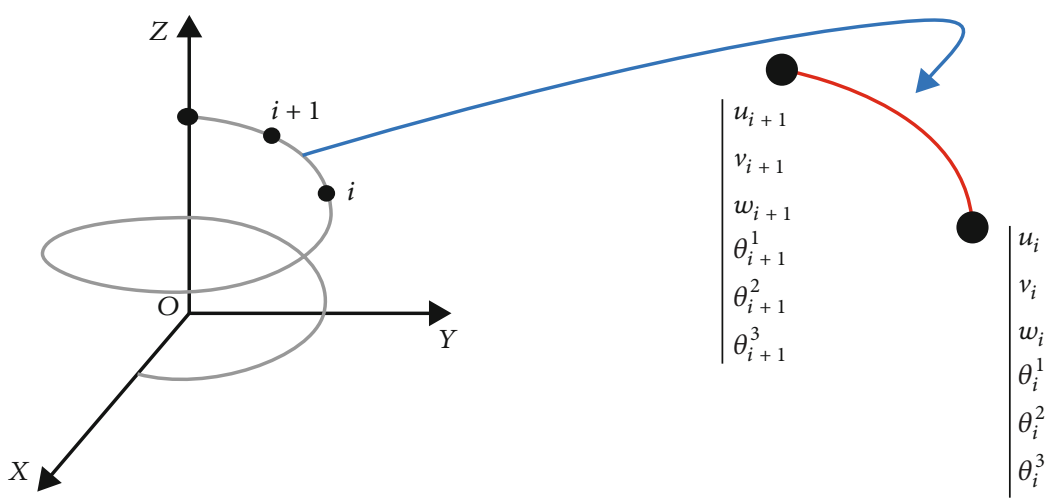

FIGURE 3: Discretization of the middle line into curvilinear finite elements.

The interpolation of the displacement vector in each element is given by

$$
\left\{\begin{array}{l}
u^{e}(s)=N_{1}(s) u_{i}+N_{2}(s) u_{i+1} ; \theta^{1 e}(s)=N_{1}(s) \theta_{i}^{1}+N_{2}(s) \theta_{i+1}^{1} \\
v^{e}(s)=N_{1}(s) v_{i}+N_{2}(s) v_{i+1} ; \theta^{2 e}(s)=N_{1}(s) \theta_{i}^{2}+N_{2}(s) \theta_{i+1}^{2} \\
w^{e}(s)=N_{1}(s) w_{i}+N_{2}(s) w_{i+1} ; \theta^{3 e}(s)=N_{1}(s) \theta_{i}^{3}+N_{2}(s) \theta_{i+1}^{3}
\end{array}\right.
$$

where the interpolation functions are given by

$$
\begin{aligned}
& N_{1}(s)=\frac{s_{i+1}-s}{L_{e}}, \\
& N_{2}(s)=\frac{s-s_{i}}{L_{e}},
\end{aligned}
$$

where $L_{e}=L /(N e-1)$ is the length of the finite element " $e$ " and $\mathrm{Ne}$ is the number of nodes.

The elementary displacement vector $\{U\}^{e}$ and elementary displacement gradient $\{\theta\}^{e}$ are given by

$$
\left\{\begin{array}{l}
\{U\}^{e}=[N N]\{q\}^{e}, \\
\{\theta\}^{e}=[G]\{q\}^{e},
\end{array}\right.
$$

where $[N N]$ and $[G]$ are, respectively, the matrix of interpolation functions and the matrix of their derivatives with respect to the curvilinear abscissa (see Appendix E).

The variations of elementary vectors are written:

$$
\left\{\begin{array}{l}
\{\delta U\}^{e}=[N N]\{\delta q\}^{e}, \\
\{\delta \theta\}^{e}=[G]\{\delta q\}^{e},
\end{array}\right.
$$

where $\{\delta q\}^{e}$ is the variation of the nodal displacement vector.
Thus, the discretized form of the global problem (17) is written as follows:

$$
\begin{gathered}
\left\{\begin{array}{l}
\sum_{e} \int_{L_{e}}\langle\delta q\rangle^{e T}[B(\theta)]\{S\} d s-\lambda P_{\text {ext }}=0, \\
\{S\}=[D][B(\theta)]\{q\}^{e},
\end{array}\right. \\
\quad \text { or }\left\{\begin{array}{l}
{[K(q)]\{q\}-\lambda\left\{F_{\text {ext }}\right\}=0,} \\
\{S\}=[D][B(\theta)]\{q\}^{e},
\end{array}\right.
\end{gathered}
$$

where $[B(\theta)]=\left[B^{l}\right]+\left[B^{n l}\right]=([H]+[A(\theta)])[G]$ and $[K(q)]$ is a matrix which depends on the unknown vector $\{q\}$.

3.2. Taylor Series Expansion. In this stage, we write the unknown vectors, as well as the loading parameter $\lambda$ in the form of Taylor series developments truncated to the order $p$, that is to say,

$$
\left\{\begin{array}{l}
\{q\}=\left\{q_{0}\right\}+\sum_{k=1}^{p} a^{k}\left\{q_{k}\right\}, \\
\lambda=\lambda_{0}+\sum_{k=1}^{p} a^{k} \lambda_{k}, \\
\{S\}=\left\{S_{o}\right\}+\sum_{k=1}^{p} a^{k}\left\{S_{k}\right\},
\end{array}\right.
$$

where $\left\{q_{0}\right\},\left\{S_{0}\right\}$, and $\lambda_{0}$ are known solutions at the initial point and " $a$ " is the parameter of the asymptotic development defined by [15]

$$
\left(\langle q\rangle-\left\langle q_{0}\right\rangle\right)\left\{q_{1}\right\}+\left(\lambda-\lambda_{0}\right) \lambda_{1}=a
$$

By injecting the developments (24) into (23) and (25) and by identifying the coefficients according to the different 
TABLE 1: Mechanical and geometric characteristics of the studied structure.

\begin{tabular}{lcccccc}
\hline Young's modulus & Poisson's ratio & Middle radius & Radius of circular section & Pitch of helix & Number of coils & Length of the structure \\
\hline$E=2.110^{11} \mathrm{~Pa}$ & $v=0.28$ & $R=0.5 \mathrm{~m}$ & $r_{\mathrm{o}}=0.075 \mathrm{~m}$ & $h=0.19 \mathrm{~m}$ & $N_{\mathrm{c}}=2$ & $L=6.2947 \mathrm{~m}$ \\
\hline
\end{tabular}

powers of the parameter " $a$," we obtain a succession of linear problems given as follows:

Problem at order $k=1$ :

$$
\left\{\begin{array}{l}
{\left[K_{t}\right]\left\{q_{1}\right\}=\lambda_{1}\left\{F_{\text {ext }}\right\}} \\
\left\{S_{1}\right\}=[D]\left[B\left(\theta_{0}\right)\right]\left\{q_{1}\right\} \\
\left\langle q_{1}\right\rangle\left\{q_{1}\right\}+\lambda_{1}^{2}=1
\end{array}\right.
$$

Problem at order $2 \leq k \leq p$ :

$$
\left\{\begin{array}{l}
{\left[K_{t}\right]\left\{q_{k}\right\}=\lambda_{k}\left\{F_{\mathrm{ext}}\right\}+\left\{F_{k}^{n l}\right\},} \\
\left\{S_{k}\right\}=[D]\left[B\left(\theta_{0}\right)\right]\left\{q_{k}\right\}^{e}+\left\{S_{k}^{n l}\right\}, \\
\left\langle q_{1}\right\rangle\left\{q_{k}\right\}+\lambda_{1} \lambda_{k}=0,
\end{array}\right.
$$

where the tangent stiffness matrix $\left[K_{t}\right]$ of the discretized structure, evaluated at the initial point, is given by

$$
\left[K_{t}\right]=\sum_{e} \int_{L_{e}}\left(\left[B\left(\theta_{0}\right)\right]^{t}[D]\left[B\left(\theta_{0}\right)\right]+[G]^{t}\left[\widehat{S}_{0}\right][G]\right) d s .
$$

$\left\{F_{k}^{n l}\right\}$ is a vector which depends on solutions to the previous orders, given by

$$
\left\{F_{k}^{n l}\right\}=-\sum_{e} \int_{L_{e}}\left(\sum_{r=1}^{k-1}\left[B_{r}^{n l}\right]^{t}\left\{S_{k-r}\right\}+\frac{1}{2}\left[B_{0}\right]^{t}[D] \sum_{r=1}^{k-1}\left[A\left(\theta_{r}\right]\left\{\theta_{k-r}\right\}\right) d s,\right.
$$

and $\left[\widehat{S}_{0}\right]$ is the matrix of initial stresses (see Appendix F).

Thus, the computation of each asymptotic branch of the nonlinear problem (23) requires a single inversion of the tangent matrix. The validity range of the series (24) is defined by the following criterion [17]:

$$
a_{\max }=\left(\varepsilon \frac{\left\|U_{1}\right\|}{\left\|U_{p}\right\|}\right)^{1 /(p-1)}
$$

where $\varepsilon$ is the tolerance parameter.

3.3. Continuation Technique. For the computation of the entire solution of the nonlinear problem (23), we apply the continuation technique [17] which consists in updating the

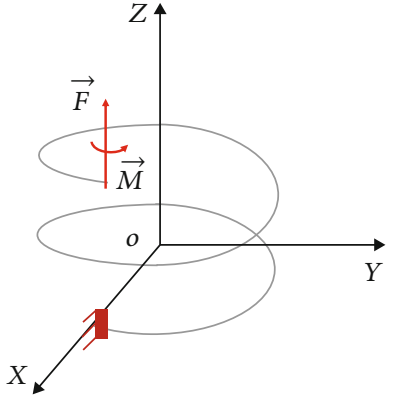

FIGURE 4: Recessed-loaded helical structure.

tangent matrix (28) at the new initial point defined by $\left\{q_{0}\right\}$ $=\left\{q\left(a_{\max }\right)\right\},\left\{S_{0}\right\}=\left\{S\left(a_{\max }\right)\right\}$, and $\lambda_{0}=\lambda\left(a_{\max }\right)$.

\section{Results and Discussions}

In this part, we consider a helical structure whose mechanical and geometric characteristics are mentioned in Table 1. One of the ends of this structure is embedded, the other end is subjected to external actions represented by the torsor $\left\{F=10^{3} \mathrm{~N} ; M=50 \mathrm{~N} \cdot \mathrm{m}\right\}$ as shown in Figure 4 .

Using this example, we solve the nonlinear problem (23) by the proposed high-order algorithm and the NewtonRaphson algorithm. The calculated displacements are expressed in the Cartesian coordinate system and the rotations in the Frenet coordinate system. In this application, we first study the influence of the mesh and the truncation order of the Taylor series on the solution quality of the problem (23) expressed here by the decimal logarithm of the norm of the residual vector (see Figures 5 and 6). For that, we have chosen in the first case a truncation order $p=10$ and in the second case a mesh with 59 elements and this by using the same tolerance parameter $\varepsilon=10^{-10}$.

As shown in Figure 5, the optimal mesh corresponds to $N_{e}=60$ which ensures a good quality of the solution of the considered problem, expressed here by $(\log )_{10}\|\vec{R} e s\| \leq-6$. Figure 6 shows that if we use a mesh with 59 elements in the proposed algorithm, then the optimal truncation order corresponds well to the value $p=10$ which ensures the same quality of the solution represented in Figure 5.

In the following, we are interested in the comparison of computation times of CPU used by the high-order algorithm and the iterative incremental algorithm of Newton-Raphson and this for various meshes as shown in Table 2. In this numerical experiment, we have fixed the number of ANM steps, that is to say, $N_{\text {step }}=10$.

As shown in this table, the use of the Newton-Raphson algorithm requires for each mesh a high number of inversions of the tangent matrix in comparison with the proposed algorithm. 


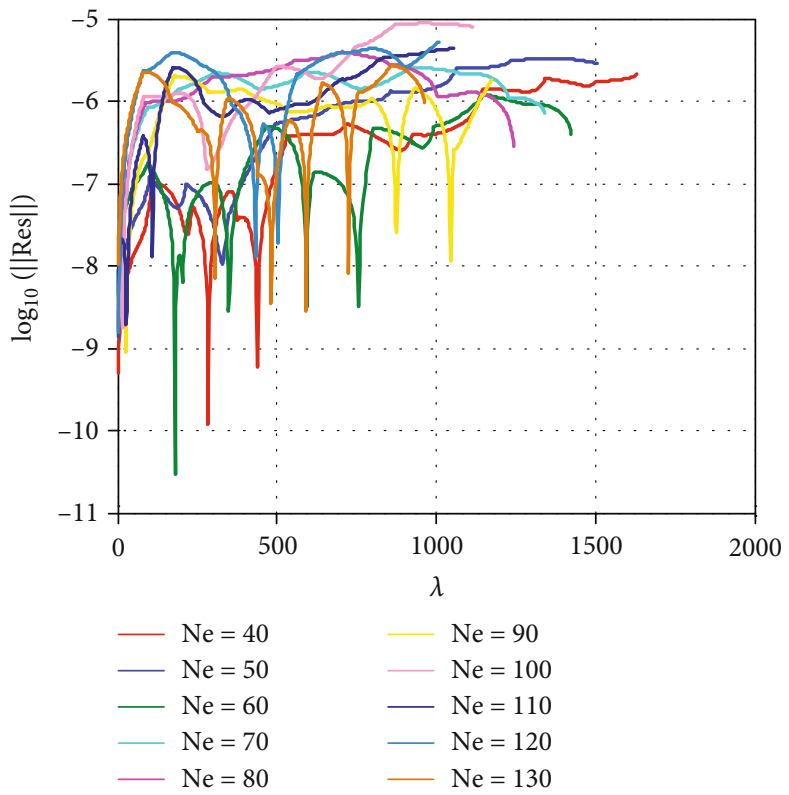

Figure 5: Influence of the mesh on the residue vector: evolution of the Euclidian norm of residue vector with respect to the loading parameter for different meshes.

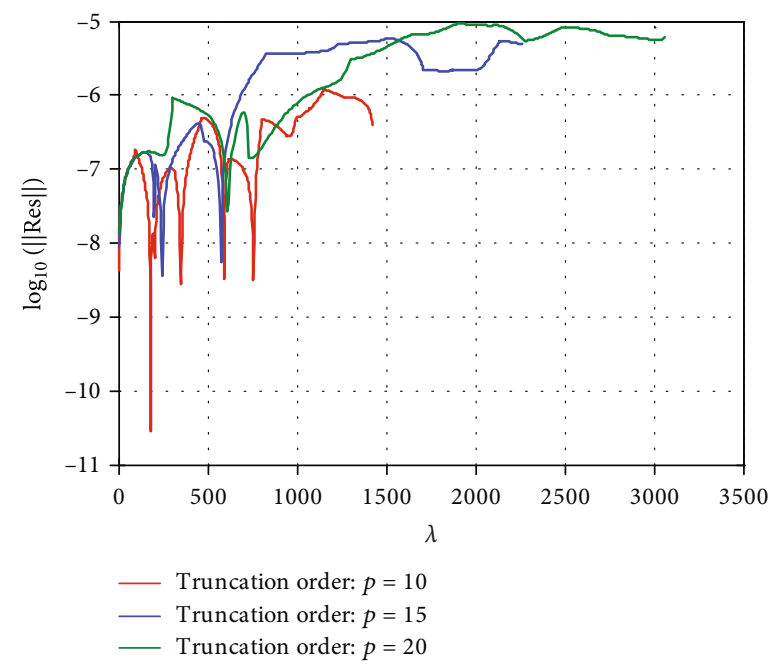

Figure 6: Influence of the truncation order of the Taylor series on the residue vector: evolution of the Euclidian norm of residue vector with respect to the loading parameter for different truncation orders.

In this application, we also studied the variation of the degrees of freedom $U_{x}, U_{y}, U_{z}, \theta^{1}, \theta^{2}$, and $\theta^{3}$ of the loaded node according to the parameter of loading $\lambda$ by using the following data: $\mathrm{Ne}=60, p=10, N_{\text {step }}=10$, and $\varepsilon=10^{-10}$ for the proposed algorithm and $\varepsilon=10^{-4}$ for the NewtonRaphson algorithm.

From Figures 7 and 8, we note the good concordance between the numerical solutions obtained by our highorder modeling and those calculated by the iterative Newton-Raphson algorithm.

In Figure 9, we represent the initial state and the middle line deformation of the considered structure, and in Figure 10, we give the evolution of the solution quality of the solved problem according to the loading parameter by using both algorithms.

From Figure 10, we note that our numerical modeling based on the ANM allows us to calculate the displacements and rotations of the middle line of the considered structure with a better quality compared to the results obtained by the Newton-Raphson algorithm.

\section{Conclusion}

In this work, we propose a high-order numerical modeling for the nonlinear elastic computation of a thin structure of helical type without neglecting any nonlinear term for the first time. 
TABLE 2: Influence of the mesh on the CPU computation time used by high-order and Newton-Raphson algorithms for different meshes and for truncation order $p=10$.

\begin{tabular}{lcccccccccc}
\hline Mesh & $\begin{array}{c}\text { Maximum } \\
\text { load }\end{array}$ & $\begin{array}{c}\text { Truncation order } \\
p\end{array}$ & Tolerance $\varepsilon$ & $\begin{array}{c}\text { Number of } \\
\text { inversions }\end{array}$ & $\begin{array}{c}\text { ANM } \\
\text { CPolerance } \\
E\end{array}$ & $\begin{array}{c}\text { NR } \\
\text { Number of } \\
\text { inversions }\end{array}$ & $\begin{array}{c}\text { CPU } \\
\text { CPU(MAN) }\end{array}$ \\
\hline $\mathrm{Ne}=40$ & 1631 & 10 & $10^{-10}$ & 10 & 3.98 & $10^{-4}$ & 3264 & 160.07 & 40.21 \\
$\mathrm{Ne}=50$ & 1505 & 10 & $10^{-10}$ & 10 & 4.73 & $10^{-4}$ & 3012 & 153.48 & 32.44 \\
$\mathrm{Ne}=60$ & 1425 & 10 & $10^{-10}$ & 10 & 5.69 & $10^{-4}$ & 2852 & 152.48 & 26.79 \\
$\mathrm{Ne}=70$ & 1342 & 10 & $10^{-10}$ & 10 & 6.48 & $10^{-4}$ & 2686 & 150.34 & 23.20 \\
$\mathrm{Ne}=80$ & 1246 & 10 & $10^{-10}$ & 10 & 7.49 & $10^{-4}$ & 2497 & 149.77 & 19.99 \\
$\mathrm{Ne}=90$ & 1176 & 10 & $10^{-10}$ & 10 & 8.31 & $10^{-4}$ & 2354 & 147.50 & 17.74 \\
$\mathrm{Ne}=100$ & 1114 & 10 & $10^{-10}$ & 10 & 9.40 & $10^{-4}$ & 2230 & 145.30 & 15.52 \\
$\mathrm{Ne}=110$ & 1056 & 10 & $10^{-10}$ & 10 & 9.36 & $10^{-4}$ & 2114 & 144.58 & 15.44 \\
$\mathrm{Ne}=120$ & 1007 & 10 & $10^{-10}$ & 10 & 10.06 & $10^{-4}$ & 2016 & 143.35 & 14.24 \\
$\mathrm{Ne}=130$ & 964.3 & 10 & $10^{-10}$ & 10 & 10.88 & $10^{-4}$ & & 1930 & 142.98 & 13.14 \\
\hline
\end{tabular}
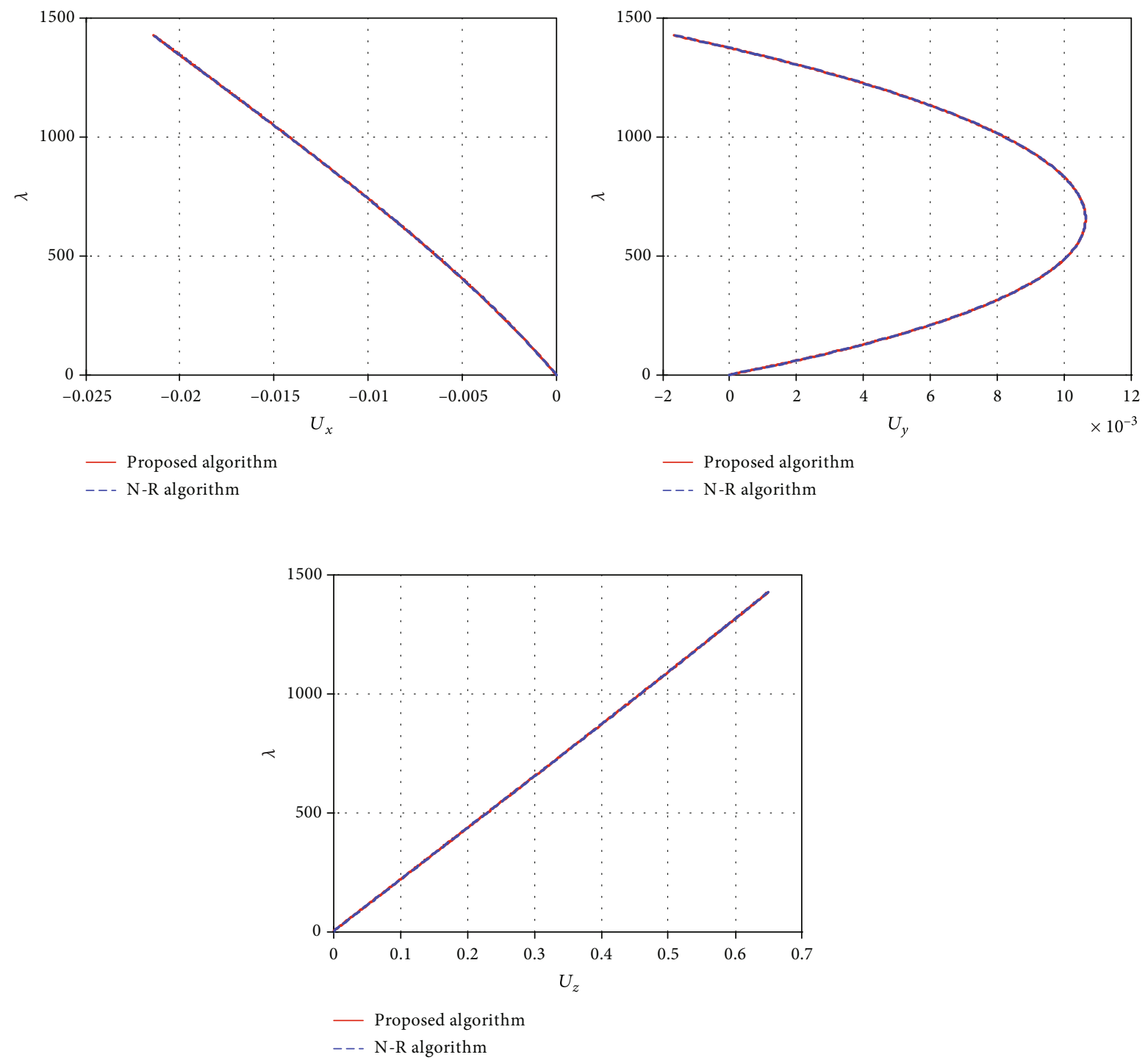

FIGURE 7: Load-displacement curves obtained by both algorithms. 

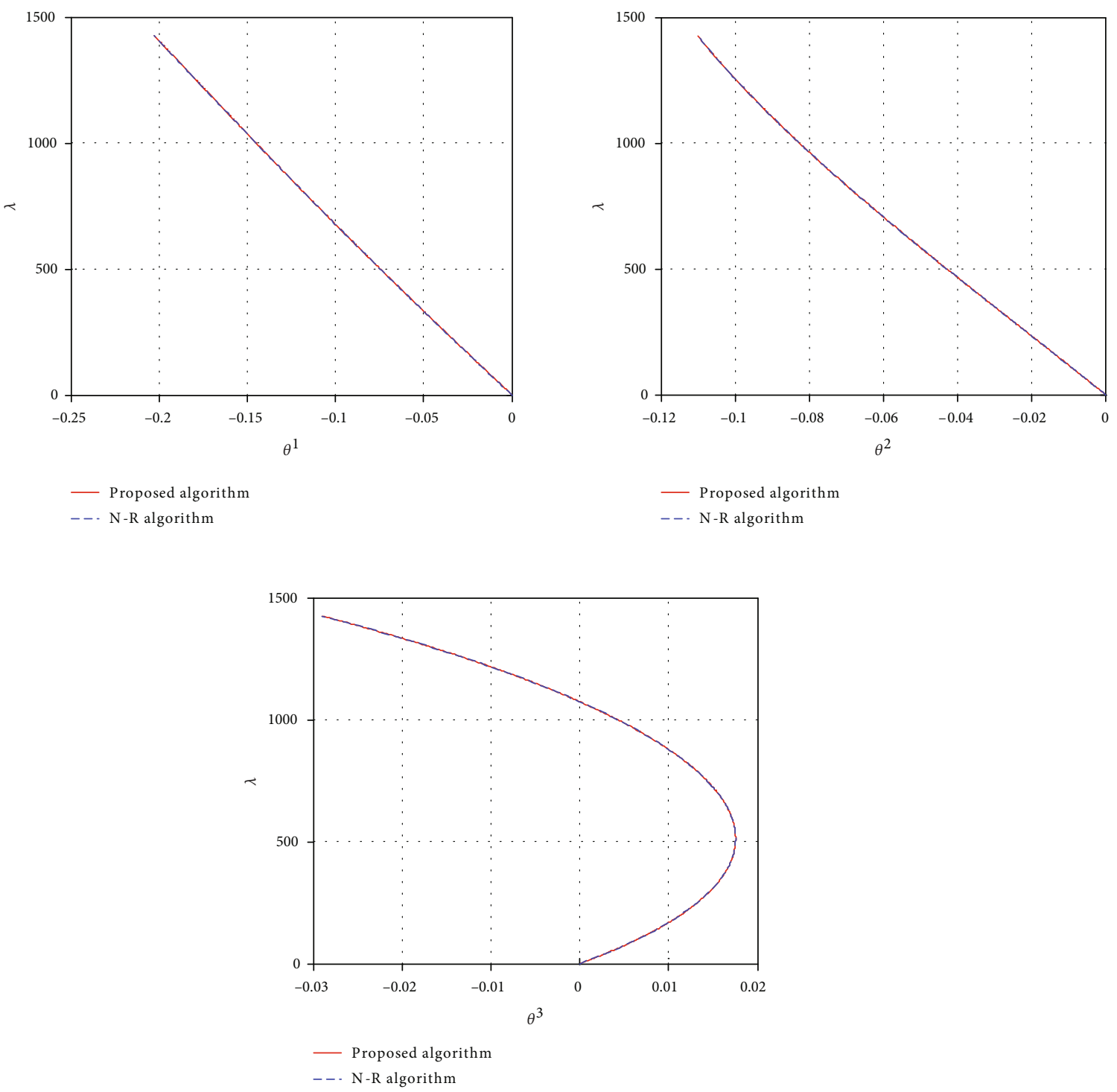

FiguRE 8: Load-rotation curves obtained by both algorithms.

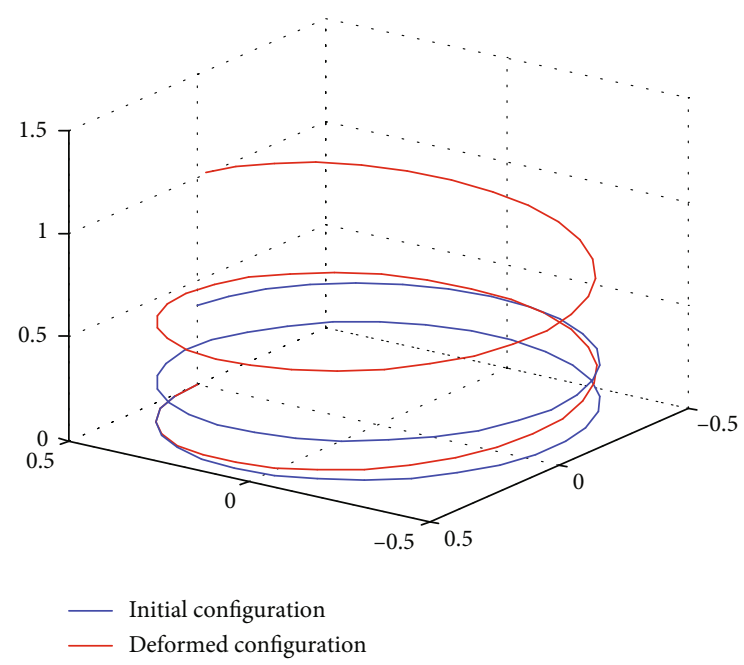

Figure 9: Initial and deformed configuration of the middle line obtained using the ANM algorithm.

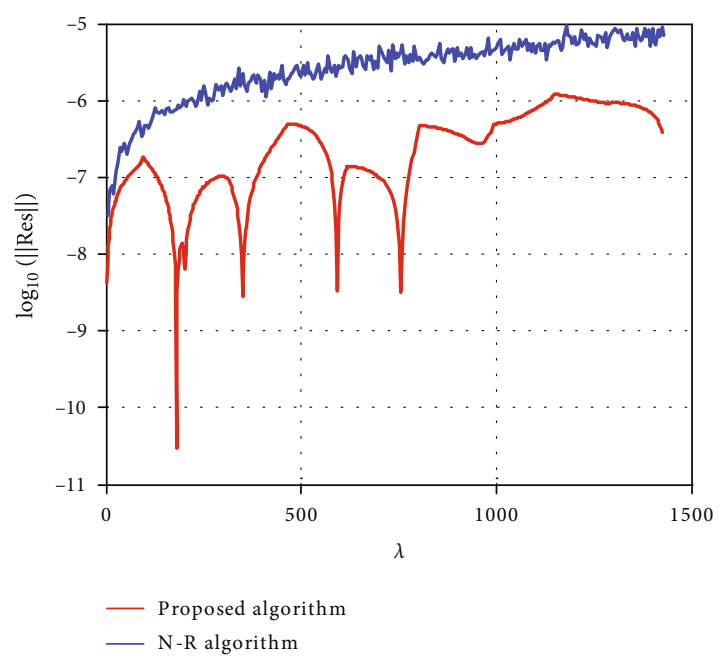

FIgURE 10: Decimal logarithm of the norm of the residue vector according to the loading parameter obtained by both algorithms. 
The algorithm realized in this context is based on the asymptotic numerical method steps. The finite element used in the discretization of the middle line of the structure considered is of curvilinear type with twelve degrees of freedom. Using a simple example, we have shown the efficiency of our numerical approach in comparison with the Newton-Raphson incremental iterative algorithm. First, we discussed the optimum choice of the mesh and the truncation order of the series allowing having the solution of the chosen example with a better quality. In the second stage, we presented a numerical analysis showing that the proposed high-order algorithm significantly reduces the number of inversions of the tangent matrix, and thus the CPU computation time, to have the entire solution of the chosen problem and this in comparison with the reference algorithm mentioned previously.

\section{Appendix}

\section{A. Generalized Deformations}

$$
\begin{aligned}
& \left(e_{t 1}=\frac{1}{2}\left(\begin{array}{c}
u_{, s}^{2}-2 \rho_{1} v u_{, s}+\left(\rho_{1} v\right)^{2}+v_{, s}^{2}+2 \rho_{1} u v_{, s}-2 \rho_{2} w v_{, s}+\left(\rho_{1} u\right)^{2} \\
-2 \rho_{2} \rho_{1} w u+\left(\rho_{2} w\right)^{2}+w_{, s}^{2}+2 \rho_{2} v w_{, s}+\left(\rho_{2} v\right)^{2}
\end{array}\right)+u_{, s}-\rho_{1} v\right. \\
& \gamma_{t n}=\frac{1}{2}\left(2 \theta^{1} w_{, s}+2 \rho_{2} v \theta^{1}-2 \theta^{3} u_{, s}+2 \rho_{1} v \theta^{3}\right)+\left(v_{, s}+\rho_{1} u-\rho_{2} w-\theta^{3}\right), \\
& \gamma_{t b}=\frac{1}{2}\left(2 \theta^{2} u_{, s}-2 \rho_{1} v \theta^{2}-2 \theta^{1} v_{, s}-2 \rho_{1} u \theta^{1}+2 \rho_{2} w \theta^{1}\right)+\left(w_{, s}+\rho_{2} v+\theta^{2}\right), \\
& \chi_{t 1}=\frac{1}{2}\left(2 \theta^{1} \theta_{, s}^{1}+2 \theta^{3} \theta_{, s}^{3}+2 \rho_{2} \theta^{2} \theta^{3}\right) \\
& \chi_{t 2}=\frac{1}{2}\left(-2 \theta^{3} \theta_{s}^{2}-2 \rho_{1} \theta^{1} \theta^{3}+2 \rho_{2} \theta_{3}^{2}\right)+\left(\theta_{, s}^{1}-\rho_{1} \theta^{2}\right), \\
& \chi_{t 3}=\frac{1}{2}\left(-2 \theta^{2} \theta_{, s}^{3}-2 \rho_{2} \theta_{, s}^{2}+2 \rho_{1} \theta^{3} \theta^{1}\right)+\left(\theta_{, s}^{1}-\rho_{1} \theta^{2}\right), \\
& \chi_{t 4}=\frac{1}{2}\left(2 \theta^{2} \theta_{, s}^{2}-2 \rho_{2} \theta^{3} \theta^{2}+2 \theta^{1} \theta_{s}^{1}\right) \\
& \chi_{n}=\frac{1}{2}\left(\begin{array}{c}
2 u_{s} \theta_{s}^{2}+2 \rho_{1} \theta^{1} u_{s}-2 \rho_{2} \theta^{3} u_{, s}-2 \rho_{1} v \theta_{, s}^{2}-2 \rho_{1}^{2} \theta^{1} v+2 \rho_{2} \rho_{1} \theta^{3} v \\
-2 \theta_{, s}^{1} v_{s}+2 \rho_{1} \theta^{2} v_{s}-2 \rho_{1} u \theta_{, s}^{1}+2 \rho_{2} w \theta_{, s}^{1}+2 \rho_{1}^{2} \theta^{2} u-2 \rho_{1} \rho_{2} \theta^{2} w
\end{array}\right)+\left(\theta_{, s}^{2}+\rho_{1} \theta^{1}-\rho_{2} \theta^{3}\right), \\
& \chi_{b}=\frac{1}{2}\left(\begin{array}{c}
2 u_{, s} \theta_{s}^{3}+2 \rho_{2} \theta^{2} u_{, s}-2 \rho_{1} v \theta_{, s}^{3}-2 \rho_{2} \rho_{1} \theta^{2} v+2 \rho_{1} \theta^{3} v_{, s}+2 \rho_{1}^{2} \theta^{3} u-2 \rho_{1} \rho_{2} \theta^{3} w \\
-2 \theta_{, s}^{1} w_{, s}-2 \rho_{2} v \theta_{, s}^{1}-\rho_{1} u_{, s}^{2}+2 \rho_{1}^{2} v u_{, s}-\rho_{1} v_{, s}^{2}-2 \rho_{1}^{2} u v_{s,}+2 \rho_{2} \rho_{1} w v_{, s}-\rho_{1}^{3} u^{2} \\
+2 \rho_{2} \rho_{1}^{2} w u-\rho_{1}\left(\rho_{2} v\right)^{2}-\rho_{1} w_{, s}^{2}-2 \rho_{2} \rho_{1} v w_{, s}-\rho_{1}\left(\rho_{2}^{2}-\rho_{1}^{2}\right) v^{2}
\end{array}\right)+\left(\theta_{, s}^{3}+\rho_{2} \theta^{2}\right), \\
& e_{t 2}=\frac{1}{2}\left(\begin{array}{c}
\left(\theta_{s,}^{3}\right)^{2}+2 \rho_{2} \theta^{2} \theta_{, s}^{3}+\left(\rho_{2} \theta^{2}\right)^{2}+\left(\rho_{1} \theta^{3}\right)^{2}+\left(\theta_{s,}^{1}\right)^{2}-2 \rho_{1} u_{s} \theta_{, s}^{3}-2 \rho_{2} \rho_{1} \theta^{2} u_{s,} \\
+2 \rho_{1}^{2} v \theta_{, s}^{3}+2 \rho_{2} \rho_{1}^{2} \theta^{2} v-2 \rho_{1}^{2} \theta^{3} v_{s}-2 \rho_{1}^{3} \theta^{2} u+2 \rho_{1}^{2} \rho_{2} \theta^{3} w+2 \rho_{1} \theta_{, s}^{1} w_{, s}+2 \rho_{2} \rho_{1} v \theta_{, s}^{1}
\end{array}\right), \\
& e_{t 3}=\frac{1}{2}\left(\left(\theta_{, s}^{2}\right)^{2}+2 \rho_{1} \theta^{1} \theta_{, s}^{2}-2 \rho_{2} \theta^{3} \theta_{, s}^{2}+\left(\rho_{1} \theta^{1}\right)^{2}-2 \rho_{2} \rho_{1} \theta^{3} \theta^{1}+\left(\rho_{2} \theta^{3}\right)^{2}+\theta_{1, s}^{2}-2 \rho_{1} \theta^{2} \theta_{, s}^{1}+\left(\rho_{1} \theta^{2}\right)^{2}\right) \text {, }
\end{aligned}
$$

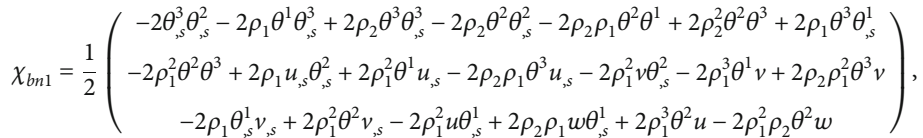

$$
\begin{aligned}
& \chi_{b n 2}=\frac{1}{2}\left(\rho_{1}\left(\theta_{s}^{3}\right)^{2}+2 \rho_{1} \rho_{2} \theta^{2} \theta_{s}^{3}+\rho_{1}\left(\rho_{2} \theta^{2}\right)^{2}+\rho_{1}\left(\rho_{1} \theta^{3}\right)^{2}+\rho_{1}\left(\theta_{, s}^{1}\right)^{2}\right) \\
& \chi_{b n 3}=\frac{1}{2}\left(\begin{array}{c}
\rho_{1}\left(\theta_{, s}^{2}\right)^{2}+2 \rho_{1}^{2} \theta^{1} \theta_{, s}^{2}-2 \rho_{2} \rho_{1} \theta^{3} \theta_{s,}^{2}+\rho_{1}\left(\rho_{1} \theta^{1}\right)^{2}-2 \rho_{2} \rho_{1}^{2} \theta^{3} \theta^{1}+\rho_{1}\left(\rho_{2} \theta^{3}\right)^{2}+\rho_{1}\left(\theta_{s,}^{1}\right)^{2} \\
-2 \rho_{1}^{2} \theta^{2} \theta_{, s}^{1}+\rho_{1}\left(\rho_{1} \theta^{2}\right)^{2}
\end{array}\right) \text {, } \\
& \chi_{b n 4}=\frac{1}{2}\left(\begin{array}{c}
-2 \rho_{1} \theta_{s, s}^{3} \theta_{, s}^{2}-2 \rho_{1}^{2} \theta^{1} \theta_{, s}^{3}+2 \rho_{2} \rho_{1} \theta^{3} \theta_{s,}^{3}-2 \rho_{2} \rho_{1} \theta^{2} \theta_{, s}^{2}-2 \rho_{2} \rho_{1}^{2} \theta^{2} \theta^{1}+2 \rho_{2}^{2} \rho_{1} \theta^{2} \theta^{3} \\
+2 \rho_{1}^{2} \theta^{3} \theta_{, s}^{1}-2 \rho_{1}^{3} \theta^{2} \theta^{3}
\end{array}\right) .
\end{aligned}
$$


B. Generalized Stresses

$$
\begin{aligned}
N & =\int_{S} S_{11} d S, \\
T_{n} & =\int_{S} S_{12} d S, \\
T_{b} & =\int_{S} S_{13} d S, \\
M_{1} & =\int_{S} X_{2} S_{12} d S, \\
M_{2} & =\int_{S}-X_{3} S_{12} d S, \\
M_{3} & =\int_{S} X_{2} S_{13} d S, \\
M_{4} & =\int_{S} X_{3} S_{13} d S, \\
M_{n} & =\int_{S} X_{3} S_{11} d S, \\
M_{b} & =\int_{S}-X_{2} S_{11} d S, \\
M_{5} & =\int_{S} X_{2}^{2} S_{11} d S, \\
M_{6} & =\int_{S} X_{3}^{2} S_{11} d S, \\
M_{7} & =\int_{S} X_{2} X_{3} S_{11} d S, \\
M_{8} & =\int_{S} X_{2}^{3} S_{11} d S, \\
M_{9} & =\int_{S} X_{2} X_{3}^{2} S_{11} d S, \\
M_{10} & =\int_{S} X_{2}^{2} X_{3} S_{11} d S,
\end{aligned}
$$


C. Matrices $[H]$ and $[A(\theta)]$

$$
\begin{aligned}
& {[H]=\left[\begin{array}{cccccccccccc}
1 & 0 & 0 & 0 & 0 & 0 & 0 & -\rho_{1} & 0 & 0 & 0 & 0 \\
0 & 1 & 0 & 0 & 0 & 0 & \rho_{1} & 0 & -\rho_{2} & 0 & 0 & -1 \\
0 & 0 & 1 & 0 & 0 & 0 & 0 & \rho_{2} & 0 & 0 & 1 & 0 \\
0 & 0 & 0 & 0 & 0 & 0 & 0 & 0 & 0 & 0 & 0 & 0 \\
0 & 0 & 0 & 1 & 0 & 0 & 0 & 0 & 0 & 0 & -\rho_{1} & 0 \\
0 & 0 & 0 & 1 & 0 & 0 & 0 & 0 & 0 & 0 & -\rho_{1} & 0 \\
0 & 0 & 0 & 0 & 0 & 0 & 0 & 0 & 0 & 0 & 0 & 0 \\
0 & 0 & 0 & 0 & 1 & 0 & 0 & 0 & 0 & \rho_{1} & 0 & -\rho_{2} \\
0 & 0 & 0 & 0 & 0 & 1 & 0 & 0 & 0 & 0 & \rho_{2} & 0 \\
0 & 0 & 0 & 0 & 0 & 0 & 0 & 0 & 0 & 0 & 0 & 0 \\
0 & 0 & 0 & 0 & 0 & 0 & 0 & 0 & 0 & 0 & 0 & 0 \\
0 & 0 & 0 & 0 & 0 & 0 & 0 & 0 & 0 & 0 & 0 & 0 \\
0 & 0 & 0 & 0 & 0 & 0 & 0 & 0 & 0 & 0 & 0 & 0 \\
0 & 0 & 0 & 0 & 0 & 0 & 0 & 0 & 0 & 0 & 0 & 0 \\
0 & 0 & 0 & 0 & 0 & 0 & 0 & 0 & 0 & 0 & 0 & 0
\end{array}\right]} \\
& {[A(\theta)]=\left[\begin{array}{cccccccccccccccc}
A_{1} & A_{2} & A_{3} & 0 & 0 & 0 & \rho_{1} A_{2} & A_{17} & -\rho_{2} A_{2} & 0 & 0 & 0 \\
A_{7} & 0 & A_{8} & 0 & 0 & 0 & 0 & A_{10} & 0 & A_{3} & 0 & -A_{1} \\
-A_{7} & 0 & A_{9} & 0 & 0 & 0 & -\rho_{1} A_{9} & -\rho_{1} A_{8} & \rho_{2} A_{9} & -A_{2} & A_{1} & 0 \\
A_{8} & -A_{9} & 0 & A_{9} & 0 & A_{7} & 0 & 0 & 0 & A_{6} & \rho_{2} A_{7} & A_{11} \\
0 & 0 & 0 & 0 & A_{7} & 0 & 0 & 0 & 0 & \rho_{1} A_{7} & 0 & A_{12} \\
0 & 0 & 0 & 0 & 0 & -A_{8} & 0 & 0 & 0 & \rho_{1} A_{7} & A_{13} & \rho_{1} A_{9} \\
0 & 0 & 0 & A_{9} & A_{8} & 0 & 0 & 0 & 0 & A_{6} & A_{14} & -\rho_{2} A_{8} \\
A_{12} & 0 & A_{15} & -A_{2} & A_{1} & 0 & \rho_{1} A_{15} & -\rho_{1} A_{12} & -\rho_{2} A_{15} & \rho_{1} A_{1} & \rho_{1} A_{2} & -\rho_{2} A_{1} \\
A_{18} & A_{19} & A_{20} & -A_{3} & 0 & A_{1} & \rho_{1} A_{21} & A_{22} & A_{16} & 0 & \rho_{2} A_{1} & \rho_{1} A_{2} \\
-\rho_{1} A_{11} & -\rho_{1}^{2} A_{7} & \rho_{1} A_{6} & -A_{20} & 0 & A_{18} & -\rho_{1}^{3} A_{7} & -\rho_{1} A_{22} & \rho_{1}^{2} \rho_{2} A_{7} & 0 & \rho_{2} A_{18} & \rho_{1} A_{21} \\
0 & 0 & 0 & -A_{15} & A_{12} & 0 & 0 & 0 & 0 & \rho_{1} A_{12} & \rho_{1} A_{15} & -\rho_{2} A_{12} \\
\rho_{1} A_{12} & \rho_{1} A_{15} & 0 & A_{21} & -A_{18} & -A_{12} & \rho_{1}^{2} A_{15} & -\rho_{1}^{2} A_{12} & -\rho_{1} \rho_{2} A_{15} & -\rho_{1} A_{18} & A_{23} & A_{24} \\
0 & 0 & 0 & \rho_{1} A_{6} & 0 & \rho_{1} A_{11} & 0 & 0 & 0 & 0 & \rho_{1} \rho_{2} A_{11} & \rho_{1}^{3} A_{7} \\
0 & 0 & 0 & -\rho_{1} A_{15} & \rho_{1} A_{12} & 0 & 0 & 0 & 0 & \rho_{1}^{2} A_{12} & \rho_{1}^{2} A_{15} & -\rho_{1} \rho_{2} A_{12} \\
0 & 0 & 0 & \rho_{1}^{2} A_{7} & -\rho_{1} A_{11} & -\rho_{1} A_{12} & 0 & 0 & 0 & -\rho_{1}^{2} A_{11} & A_{25} & A_{26}
\end{array}\right]}
\end{aligned}
$$


with

where

$$
\begin{aligned}
& A_{1}=u_{, s}-\rho_{1} v, \\
& A_{2}=v_{, s}-\rho_{2} w+\rho_{1} u, \\
& A_{3}=w_{, s}+\rho_{2} v, \\
& A_{4}=\theta_{, s}^{3} \\
& A_{5}=\theta_{, s}^{2} \\
& A_{6}=\theta_{, s}^{4} \\
& A_{7}=\theta^{3}, \\
& A_{8}=\theta^{2}, \\
& A_{9}=\theta^{1}, \\
& A_{10}=\rho_{2} A_{9}+\rho_{1} A_{7}, \\
& A_{11}=A_{4}+\rho_{2} A_{8}, \\
& A_{12}=A_{5}+\rho_{1} A_{9}-\rho_{2} A_{7}, \\
& A_{13}=-A_{4}-\rho_{1} A_{8}, \\
& A_{14}=A_{5}-\rho_{2} A_{7}, \\
& A_{15}=-A_{6}+\rho_{1} A_{8}, \\
& A_{16}=-\rho_{2} A_{21}, \\
& A_{17}=\rho_{2} A_{3}-\rho_{1} A_{1}, \\
& A_{18}=A_{11}-\rho_{1} A_{1}, \\
& A_{19}=-\rho_{1}\left(A_{1}+A_{7}\right), \\
& A_{20}=-\rho_{1} A_{3}-A_{6}, \\
& A_{21}=-\rho_{1}\left(A_{2}+A_{7}\right), \\
& A_{22}=-\rho_{1} A_{11}-\rho_{2} A_{6}, \\
& A_{23}=\rho_{1}^{2} A_{2}-\rho_{2} A_{12}-\rho_{1}^{2} A_{7}, \\
& A_{24}=\rho_{2} A_{11}-\rho_{1} A_{15}-\rho_{2} \rho_{1} A_{1}, \\
& A_{25}=-\rho_{2} \rho_{1} A_{12}-\rho_{1}^{3} A_{7}, \\
& A_{26}=\rho_{2} \rho_{1} A_{11}-\rho_{1}^{3} A_{7}, \\
&
\end{aligned}
$$

\section{Matrix $[D]$}

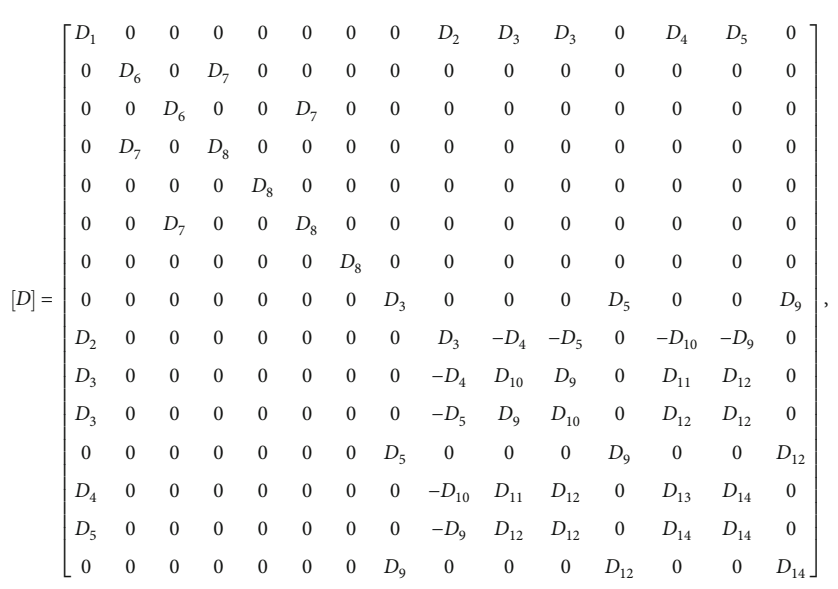

with

$$
\begin{aligned}
& I_{1}=\int\left(X_{2}^{2}+X_{3}^{2}\right) d S=\frac{\pi r^{4}}{2}, \\
& I_{2}=\int X_{3}^{2} d S=I_{3}=\int X_{2}^{2} d S==\frac{\pi r^{4}}{4}, \\
& a_{1}=\int X_{2}^{2} X_{3}^{2} d S=\frac{\pi r^{6}}{24}, \\
& a_{2}=\int X_{2}^{4} d S=\frac{\pi r^{6}}{8}, \\
& a_{3}=\int X_{2}^{6} d S=\frac{5 \pi r^{8}}{64}, \\
& a_{4}=\int X_{2}^{2} X_{3}^{4} d S=\frac{\pi r^{8}}{64} .
\end{aligned}
$$

\section{E. Matrices $[N N]$ and $[G]$}

$$
[N N]=\left[\begin{array}{cccccccccccc}
N_{1} & 0 & 0 & 0 & 0 & 0 & N_{2} & 0 & 0 & 0 & 0 & 0 \\
0 & N_{1} & 0 & 0 & 0 & 0 & 0 & N_{2} & 0 & 0 & 0 & 0 \\
0 & 0 & N_{1} & 0 & 0 & 0 & 0 & 0 & N_{2} & 0 & 0 & 0 \\
0 & 0 & 0 & N_{1} & 0 & 0 & 0 & 0 & 0 & N_{2} & 0 & 0 \\
0 & 0 & 0 & 0 & N_{1} & 0 & 0 & 0 & 0 & 0 & N_{2} & 0 \\
0 & 0 & 0 & 0 & 0 & N_{1} & 0 & 0 & 0 & 0 & 0 & N_{2}
\end{array}\right],
$$




$$
[G]=\left[\begin{array}{cccccccccccc}
\frac{d N_{1}}{d s} & 0 & 0 & 0 & 0 & 0 & \frac{d N_{2}}{d s} & 0 & 0 & 0 & 0 & 0 \\
0 & \frac{d N_{1}}{d s} & 0 & 0 & 0 & 0 & 0 & \frac{d N_{2}}{d s} & 0 & 0 & 0 & 0 \\
0 & 0 & \frac{d N_{1}}{d s} & 0 & 0 & 0 & 0 & 0 & \frac{d N_{2}}{d s} & 0 & 0 & 0 \\
0 & 0 & 0 & \frac{d N_{1}}{d s} & 0 & 0 & 0 & 0 & 0 & \frac{d N_{2}}{d s} & 0 & 0 \\
0 & 0 & 0 & 0 & \frac{d N_{1}}{d s} & 0 & 0 & 0 & 0 & 0 & \frac{d N_{2}}{d s} & 0 \\
N_{1} & 0 & 0 & 0 & 0 & 0 & N_{2} & 0 & 0 & 0 & 0 & 0 \\
0 & N_{1} & 0 & 0 & 0 & 0 & 0 & N_{2} & 0 & 0 & 0 & 0 \\
0 & 0 & N_{1} & 0 & 0 & 0 & 0 & 0 & N_{2} & 0 & 0 & 0 \\
0 & 0 & 0 & N_{1} & 0 & 0 & 0 & 0 & 0 & N_{2} & 0 & 0 \\
0 & 0 & 0 & 0 & N_{1} & 0 & 0 & 0 & 0 & 0 & N_{2} & 0 \\
0 & 0 & 0 & 0 & 0 & N_{1} & 0 & 0 & 0 & 0 & 0 & N_{2}
\end{array}\right] .
$$

\section{F. Matrix $\left[\widehat{S}_{0}\right]$}

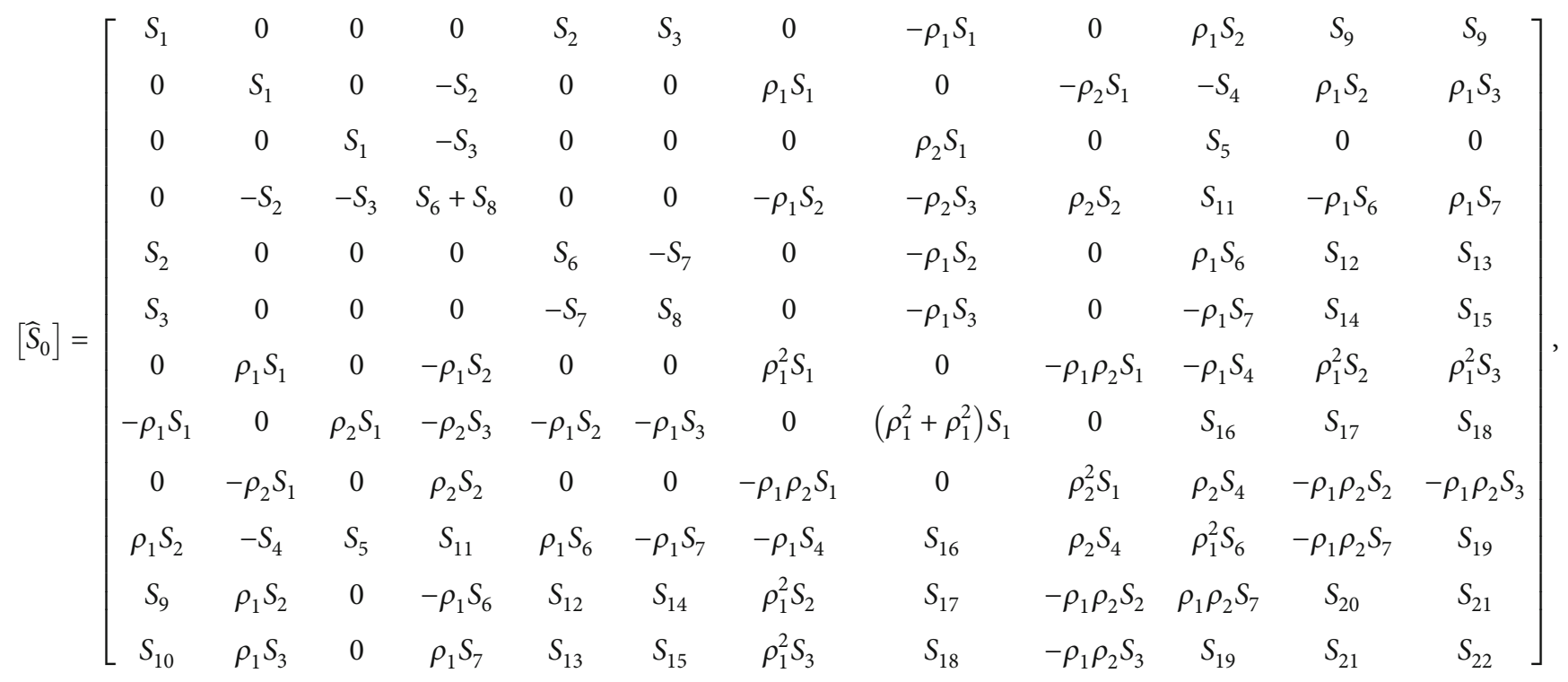


with

$$
\begin{aligned}
& S_{1}=\bar{S}(1)-\rho_{1} \bar{S}(9), \\
& S_{2}=\bar{S}(8)+\rho_{1} \bar{S}(12), \\
& S_{3}=\bar{S}(9)-\rho_{1} \bar{S}(10) \text {, } \\
& S_{4}=\bar{S}(3) \text {, } \\
& S_{5}=\bar{S}(2) \text {, } \\
& S_{6}=\bar{S}(11)+\rho_{1} \bar{S}(14), \\
& S_{7}=\bar{S}(12)-\rho_{1} \bar{S}(15) \text {, } \\
& S_{8}=\bar{S}(10)+\rho_{1} \bar{S}(13) \text {, } \\
& S_{9}=S_{4}+\rho_{1} S_{3} \text {, } \\
& S_{10}=-S_{5}-\rho_{2} S_{6} \text {, } \\
& S_{11}=\bar{S}(4)+\bar{S}(7) \text {, } \\
& S_{12}=\bar{S}(7)-\rho_{2} S_{7} \text {, } \\
& S_{13}=\bar{S}(5)-\rho_{2} S_{6} \text {, } \\
& S_{14}=-\bar{S}(6)+\rho_{2} S_{8} \text {, } \\
& S_{15}=\bar{S}(4)+\rho_{2} S_{7} \text {, } \\
& S_{16}=\rho_{2} S_{6}-\rho_{1}^{2} S_{2} \text {, } \\
& S_{17}=-\rho_{1} S_{4}-\rho_{1} \rho_{2} S_{3} \text {, } \\
& S_{18}=\rho_{1} S_{5}+\rho_{1} \rho_{2} S_{2} \text {, } \\
& S_{19}=-\rho_{1} \rho_{2} S_{3}+\rho_{1}(\bar{S}(6)+\bar{S}(5)) \text {, } \\
& S_{20}=-\rho_{1} \bar{S}(6)+\rho_{1}^{2} S_{6}+\rho_{2}^{2} S_{8} \text {, } \\
& S_{21}=\rho_{2}(\bar{S}(4)-\bar{S}(7))+\left(\rho_{1}^{2}-\rho_{2}^{2}\right) S_{7} \text {, } \\
& S_{22}=-\rho_{2} \bar{S}(5)+\rho_{1}^{2} S_{8}+\rho_{2}^{2} S_{6} \text {. }
\end{aligned}
$$

\section{Data Availability}

No data were used to support this study.

\section{Consent}

No individual participants were included in the study so there are no subjects for which informed consent requirements arise.

\section{Conflicts of Interest}

The authors declare that they have no conflict of interest.

\section{References}

[1] A. M. Wahl, Mechanical Springs, McGraw-Hill, New York, 2nd edition, 1963.

[2] S. P. Timoshenko, Mechanics of Materials, Chapman \& Hall, 1991.

[3] M. Taktak, F. Dammak, S. Abid, and M. Haddar, "A mixedhybrid finite element for three-dimensional isotropic helical beam analysis," International Journal of Mechanical Sciences, vol. 47, no. 2, pp. 209-229, 2005.

[4] D. Fakhreddine, T. Mohamed, A. Said, D. Abderrazek, and H. Mohamed, "Finite element method for the stress analysis of isotropic cylindrical helical spring," European Journal of Mechanics-A/Solids, vol. 24, no. 6, pp. 10681078, 2005.

[5] M. Taktak, F. Dammak, S. Abid, and M. Haddar, "A finite element for dynamic analysis of a cylindrical isotropic helical spring," Journal of Mechanics of Materials and Structures, vol. 3, no. 4, pp. 641-658, 2008.

[6] M. Taktak, K. Omheni, A. Aloui, F. Dammak, and M. Haddar, "Dynamic optimization design of a cylindrical helical spring," AppliedAcoustics, vol. 77, pp. 178-183, 2014.

[7] Y. Lin and A. P. Pisano, "General dynamic equations of helical springs with static solution and experimental verification," Journalof Applied Mechanics, vol. 54, no. 4, pp. 910-917, 1987.

[8] Y. Xiong and B. Tabarrok, "A finite element model for the vibration of spatial rods under various applied loads," International Journal of Mechanical Sciences, vol. 34, no. 1, pp. 41-51, 1992.

[9] V. Yıldırım, "Numerical buckling analysis of cylindrical helical coil springs in a dynamic manner," International Journal of Engineering \& Applied Sciences, vol. 1, no. 1, pp. 20-32, 2009.

[10] V. Yıldırım, "On the linearized disturbance dynamic equations for buckling and free vibration of cylindrical helical coil springs under combined compression and torsion," Meccanica, vol. 47, no. 4, pp. 1015-1033, 2012.

[11] I. Kacar and V. Yildirim, "Free vibration/buckling analyses of noncylindrical initially compressed helical composite springs," Mechanics Based Design of Structures and Machines, vol. 44, no. 4, pp. 340-353, 2015.

[12] N. Damil and M. Potier-Ferry, "A new method to compute perturbed bifurcations: application to the buckling of imperfect elastic structures," International Journal of Engineering Science, vol. 28, no. 9, pp. 943-957, 1990.

[13] M. Potier-Ferry, N. Damil, B. Braikat et al., "Treatment of strong non-linearities by the asymptotic numerical method," Comptes Rendus de l'Académie des Sciences - Series IIB Mechanics-Physics-Chemistry-Astronomy, vol. 324, no. 3, pp. 171-177, 1997.

[14] B. Cochelin, N. Damil, and M. Potier-Ferry, Méthode asymptotique numérique, Hermés Lavoisier, 2007.

[15] H. Mottaqui, B. Braikat, and N. Damil, "Discussion about parameterization in the asymptotic numerical method: application to nonlinear elastic shells," Computer Methods in Applied Mechanics and Engineering, vol. 199, no. 25-28, pp. 1701-1709, 2010.

[16] H. Mottaqui, B. Braikat, and N. Damil, "Local parameterization and the asymptotic numerical method," Mathematical Modelling of Natural Phenomena, vol. 5, no. 7, pp. 16-22, 2010.

[17] B. Cochelin, "A path-following technique via an asymptoticnumerical method," Computers \& Structures, vol. 53, no. 5, pp. 1181-1192, 1994.

[18] K. J. Bathe, Finite Element Procedure in Engineering Analysis, Pren- tice-Hall, Englewood Cliffs, 1982.

[19] O. C. Zienkiewicz and R. L. Taylor, The Finite Element Method, Berk Shire: Mc Graw-Hill, 1991. 
[20] O. C. Zienkiewicz, "Incremental displacement in non-linear analysis," International Journal for Numerical Methods in Engineering, vol. 3, no. 4, pp. 587-588, 1971.

[21] M. A. Crisfield, "A faster modified newton-raphson iteration," Computer Methods in Applied Mechanics and Engineering, vol. 20, no. 3, pp. 267-278, 1979. 